\title{
CCAAT/Enhancer Binding Protein Alpha
}

National Cancer Institute

\section{Source}

National Cancer Institute. CCAAT/Enhancer Binding Protein Alpha. NCI Thesaurus. Code C45488.

CCAAT/enhancer binding protein alpha (358 aa, $\sim 38 \mathrm{kD}$ ) is encoded by the human CEBPA gene. This nuclear protein is a DNA-binding bZIP transcription factor that, as a homodimer, recognizes common motifs in both the promoter (CCAAT sequence) and enhancer (core homology sequence) regions of genes. 